\title{
DYNAMIC WIND SPEED MODEL FOR SOLVING WIND POWER PROBLEMS
}

\author{
Obukhov S.G. ${ }^{1}$, Plotnikov I.A. ${ }^{1}$, Masolov V.G. ${ }^{2}$ \\ 1National Research Tomsk Polytechnic University, Tomsk, Russia, igorplt@tpu.ru \\ 2"VDM-tekhnika" LLC, Moscow, Russia,vdm-tech@mail.ru
}

\begin{abstract}
This work is devoted to the development of a dynamic model of wind speed designed to solve the problems of wind power. The time model of wind is presented in the form of two components - constant and turbulent. The Kaimal spectral model recommended by IEC 61400-1:2005 is used to describe the turbulent component of wind speed. The initial data for the calculation of turbulence parameters are the class of wind power plant, which is determined by its location, the height of the axis of rotation of the wind wheel and the average wind speed for the specified time interval of simulation. Computer implementation of wind model is carried out on the basis of statistically independent sources of white noise acting on forming filters, output signals of which are summed. The analysis of the obtained results shows that the wind flow model implemented on the basis of the method of forming filters provides adequate modeling of the longitudinal component of wind speed and can be used to solve the problems of wind power engineering.
\end{abstract}

Keywords: wind power, wind model, spectral model, forming filters.

\section{Introduction}

The priority direction of the development of modern energy is the application of the improvement of environmentally friendly technologies for the production of electric energy. Wind power is one of the most popular and widely used renewable energy technologies. According to the International Renewable Energy Agency (IRENA), about $52 \mathrm{GW}$ of wind-based generation was introduced in 2017, bringing the total installed capacity of wind power plants to about $539 \mathrm{GW}$ [1]. According to Harvard University studies based on very conservative assumptions, by 2020 the installed capacity of wind power will reach $1000 \mathrm{GW}$, and in many technologically developed countries the share of wind power will be more than $30 \%$. The rapid development of wind power is greatly facilitated by the efforts of scientists aimed at improving the technologies of component production and operation of wind power plants $[2,3]$.

One of the pressing tasks of increasing the energy efficiency of wind power plants is to find new and improve the known control algorithms [4-7]. The necessity to solve this problem is due to the fact that in order to extract the maximum available wind flow power, it is necessary to control the speed of $\mid$ wind turbine (WT) rotation according to certain laws in accordance with the dynamics of wind speed change. Given the high complexity of the problem identified, most researchers use mathematical computer modeling techniques to solve it. One of the most important components of the integrated mathematical model of WT required for research is the dynamic model of wind speed. The development of a dynamic model of wind speed is a difficult technical task due to the transient characteristics of wind speed data, which largely reflect turbulent dynamics in the atmosphere. The analysis of scientific papers on dynamic wind modelling for wind power has shown that the authors apply different techniques and methods, which differ mainly in describing the turbulent component of wind flow [8-10]. At the same time quality of wind models in this field of research is mainly determined by adequacy of display of dynamics of change of longitudinal component of wind flow and simplicity of computer implementation. 
The purpose of this research was to develop a universal dynamic wind model to solve various wind power problems implemented in the Matlab/Simulink software complex.

\section{Theoretical justification of dynamic wind model}

Modern software complexes such as ANSYS, ARIMA and others [11, 12] allow to model stationary and non-stationary turbulent air flows in time and space. The main purpose of these software complexes is to model aerodynamic effects on structural elements of high-rise buildings. The use of complex multi-dimensional wind models built on the Navier-Stokes differential equations describing the movement of non-stationary airflow for the identified study tasks appears to be redundant, and wind models based on statistical distribution functions have become most common in wind power. Of greatest interest is the longitudinal component of wind speed, which ultimately determines the mechanical and output electrical power of the WT. Time fluctuations of major meteorological factors, such as wind speed, pressure, humidity, etc., contain components ranging from fractions of a second to at least hundreds of years. From the point of view of simulating the operating modes of the WT, time intervals ranging from seconds to several hours are of practical interest.

Micrometeorological variations in wind speed with periods ranging from a fraction of a second to minutes are determined by small-scale turbulence. Its energy spectrum in the surface layer of the atmosphere has a maximum in a period of about $1 \mathrm{~min}$. In a meso-meteorological time interval with periods of minutes to hours, intense fluctuations of meteorological elements are relatively rare. It allows obtaining relatively stable average values of wind speed, temperature, etc., using averaging over periods of 10-20 min. Figure 1 shows the spectral distribution of horizontal wind speed constructed by Van der Hoven from measurements at the 125-metre meteorological tower at Brookhaven.

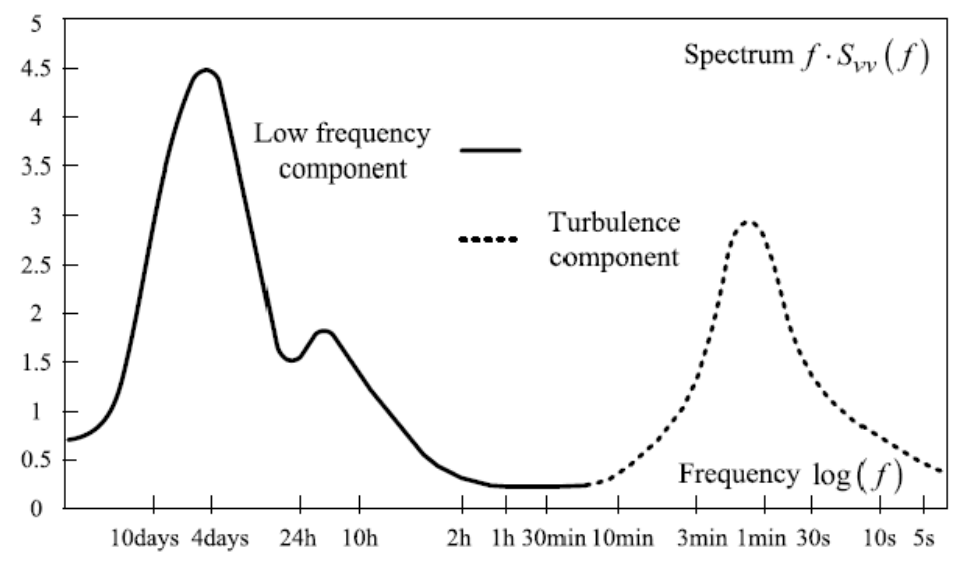

Fig.1. Horizontal wind speed spectrum

It can be seen from the graph that the frequency of the energy spectrum of the synoptic and daily fluctuations of wind speed differs significantly from the high-frequency fluctuations of turbulence, which allows to use independent time sampling for their mathematical description with subsequent superposition.

Under the assumptions taken, the time model of wind can be represented as an equation:

$V(t)=\bar{V}+v(t)$,

where $\bar{V}$ is the average speed of wind is 10 minute time interval; $v(t)$ is a dynamic or turbulent component of speed of wind.

For statistical description of the turbulent component of wind speed in wind power, empirical models of spectral density $\mathrm{S}(\mathrm{f})$ are preferably used, the most famous of which are Davenport, 
Karman and Kaimal functions [13]. To model the dynamic component of wind speed, the current work used the Kaimal spectral model recommended by the international standard [14].

According to the normal turbulence model, turbulent wind velocity fluctuations are assumed to be a stationary field of random vectors whose constituents have a Gaussian statistical distribution with zero mathematical expectation. The power spectral densities of the normalized components for the Kaimal model are described by the equation:

$$
\frac{f \cdot S(f)}{\sigma^{2}}=\frac{4 \cdot f \cdot L / \bar{V}}{(1+6 \cdot f \cdot L / \bar{V})^{5 / 3}},
$$

where $f$ is a frequency in $\mathrm{Hz} ; S(f)$ is unilateral range of a longitudinal component of a vector of speed; $\sigma$ - means square deviation of a longitudinal component of a vector of speed; $L-$ is integrated large-scale parameter of turbulence.

Spectral decomposition shows a stationary random function decomposed into harmonic oscillations of different frequencies $f_{1}, f_{2}, . . f_{k}$, with harmonics amplitudes being random values. According to Fourier's theorem any function with the period of $\pi$ can be presented in the row form:

$$
f(t)=A_{0}+\sum_{k=1}^{\infty} A_{k} \cdot \cos \left(\omega_{k} t+\varphi_{k}\right),
$$

where $A_{k}$ is the amplitude of $k$-th harmonic oscillation; $\omega_{k}$ is the circular frequency of harmonic oscillation; $\varphi_{k}$ is an initial phase $k$-th fluctuation; $A_{0}$ is the free member representing population mean of function on $\pi$ interval.

On the other hand, the dispersion of a stationary random function is equal to the sum of the dispersions of all harmonics of its spectral decomposition:

$$
D=\sigma^{2}=\int_{0}^{\infty} S(f) \cdot d f=\sum_{k=0}^{\infty} D_{k}=\sum_{k=0}^{\infty} S\left(f_{k}\right) \cdot \Delta f
$$

If the same set of frequencies is used for spectral decomposition of the function and Fourier series, it follows from (3) and (4) that the amplitude of the $k$-th harmonic oscillation of the Fourier series will be equal to the standard deviation of the corresponding harmonic of the spectrum:

$$
A_{k}=\sqrt{D_{k}}=\sqrt{S\left(f_{k}\right) \cdot \Delta f},
$$

where $\Delta f$ is the distance between adjacent frequencies.

For a final number of frequencies $N$, the equation for the longitudinal component of wind velocity determined at time interval $T$ can be represented as follows:

$$
V(t)=\bar{V}+\sum_{k=1}^{N} A_{k} \cdot \cos \left(\omega_{k} t+\varphi_{k}\right),
$$

where $\bar{V}$ is the wind speed averaged over a 10 -minute time interval.

In expression (6) time of modeling of T corresponds to a half-cycle of the main harmonica: $T=$ $\pi$, respectively number $N$ determines the frequency of sampling of a temporary signal:

$$
\Delta t=\frac{T}{N} ; \quad \Delta \omega=\frac{\pi}{T} ; \quad \Delta f=\frac{1}{2 \cdot T} ; t=k \cdot \Delta t \quad k=1 \ldots N \quad f_{k}=\frac{k}{2 \cdot T}
$$

Expressions (2) - (7) allow to construct a simulated time model of the longitudinal component of wind speed, if spectral parameters of turbulence are known. The size of spectral density for the corresponding frequency of $f_{k}$ is defined from the equation (2), and the phase corner of $\varphi_{k}$ represents a random number in the range from 0 to $2 \pi$. The spectral parameters of turbulence for the Kaimal model are determined in accordance with the requirements specified in IEC 61400-1:2005 [14], according to which all wind power plants in terms of turbulence intensity are divided into subclass A, B, C, each of which is characterized by its expected value of turbulence intensity of air flow at the height of the axis of the wind wheel $I_{\text {ref. }}$. 
Class A corresponds to WT with increased turbulence $\left(I_{\mathrm{ref}}=0.16\right)$ and is accepted for urbanized area where the roughness of the Earth's surface is $z_{0}>0.3$. Class $\mathrm{B}$ corresponds to more open area $\left(0.1<z_{0}<0.3\right)$ and turbulence intensity for it is taken equal to $I_{\mathrm{ref}}=0.14$. Class $\mathrm{C}$ is characterized by open area $\left(z_{0}<0.1\right)$ with turbulence intensity $I_{\text {ref }}=0.12$, Table 1 .

Table 1 -Basic Class Parameters WT

\begin{tabular}{|c|c|c|c|c|}
\hline Class WT & I & II & III & $S$ \\
\hline$V_{\mathrm{ref}}(\mathrm{M} / \mathrm{c})$ & 50 & 42.5 & 37.5 & \multirow{4}{*}{$\begin{array}{l}\text { Values of calculated parameters are } \\
\text { assigned by a designer }\end{array}$} \\
\hline A $\quad I_{\text {ref }}$ & \multicolumn{3}{|c|}{0.16} & \\
\hline $\mathrm{B} I_{\text {ref }}$ & \multicolumn{3}{|c|}{0.14} & \\
\hline $\mathrm{C} I_{\text {ref }}$ & \multicolumn{3}{|c|}{0.12} & \\
\hline
\end{tabular}

Wind speed in the surface layer of the atmosphere depends significantly on the roughness of the Earth's surface, consequently, the nature of the location of the WT and the height of its mast $h$ will have a direct impact on the total generation of electric energy [15]. Most meteorological stations record wind parameters at a standard height of $10 \mathrm{~m}$. To determine the average wind speed at other $V_{h}$ altitudes, an approximate empirical formula can be used, which includes the value of the standard wind speed $V_{10}$, measured at an altitude of ten meters:

$$
V_{h}=V_{10} \cdot\left(\ln \left(\frac{h}{z_{0}}\right) / \ln \left(\frac{10}{z_{0}}\right)\right), \mathrm{m} / \mathrm{s}
$$

where $z_{0}$ is the roughness of the Earth's surface, the numerical values from Table 2 [16].

$$
\Lambda=\left\{\frac{0.7 \cdot Z}{42 \mathrm{~m}} \quad \frac{Z \leq 60 \mathrm{~m}}{Z>60 \mathrm{~m}}\right.
$$

To calculate the integral scale parameter of the longitudinal component of the velocity vector $L$, the expression is used:

$$
L=8.1 \cdot \Lambda
$$

The initial data for the calculation of turbulence parameters are the WT class, which is determined by its location, the height of the axis of rotation of the wind wheel $\mathrm{Z}$ and the average wind speed for a given simulation time interval $\bar{V}_{h u b}$.

Table 2 - Parameters for calculation of vertical wind flow profile.

\begin{tabular}{|l|c|c|}
\hline \multicolumn{1}{|c|}{ Type of terrain } & Roughness of a surface, $z_{0}(\mathrm{M})$ & $\begin{array}{c}\text { Class of roughness } \\
\text { of a relief }\end{array}$ \\
\hline Water surface & 0.0001 & \\
Desert & 0.0003 & 0 \\
Flat snow cover & 0.001 & \\
Airport runway & 0.01 & 1 \\
\hline Countryside with separate buildings and & 0.05 & 2 \\
fences & 0.1 & \\
\hline Countryside with trees, forests, villages & 0.2 & 3 \\
Numerous trees, shrub & 0.3 & \\
\hline Mountain area & 0.5 & \\
The city & 1.0 & \\
Big cities, forest & & \\
\hline
\end{tabular}


The software implementation of the above model is carried out in two stages. At the first stage values of amplitudes $A_{\mathrm{k}}$ and phase corners of $\varphi_{k}$ of the corresponding harmonics of the modelled signal pay off, and at the second stage its synthesis is carried out. To fulfil the calculations, two numeric data arrays must be created: one with dimension $M 1[m, N]$, and second with $M 2[N, N t]$.

The parameter $\min$ the M1 array is determined by the number of calculated variables: $f_{\mathrm{k}}, S\left(f_{k}\right)$, $A_{k}$, etc. The value $N$ defines the number of harmonic oscillations that are taken into account in the decomposition of the function. With a small value of $N$ we obtain a low accuracy of calculation, a large value of $N$ requires additional resources. For the solved class of tasks it seems quite acceptable to choose the number of harmonics taken into account, on the order of $N=50-100$. The Nt parameter corresponds to the specified number of process calculation points used for output. It is important to note that frequency synchronization adopted in model development requires certain ratios between $N$ and $N t$.

For example: the purpose of modeling is imitation of a longitudinal component of speed of wind on a time interval of $T_{\text {mod }}=100 \mathrm{~s}$ with sampling $\Delta t=0.1 \mathrm{~s}$. If directly to accept page $T=100 \mathrm{~s}$, then at $N=50$ in expressions (7) we will receive $\Delta t=2 \mathrm{~s}$ that much more exceeds a desirable interval of sampling. For this example it is necessary to determine the decomposition period as $T=\Delta t \cdot N$, then to calculate the parameter $c$ which defines dimension of the output array $N t=\mathrm{c} \cdot N$ and number of cycles of calculation which need to be executed for its full filling:

$$
c=\frac{T_{\text {mod }}}{T}
$$

Figure 2 shows an example of simulating the longitudinal component of wind speed at a time interval of 40 seconds with a sampling of $0.1 \mathrm{~s}$. The simulation was carried out under the following conditions: average wind speed $\bar{V}=6.0 \mathrm{~m} / \mathrm{s}$, turbulence intensity $I_{\text {ref }}=0.16$, height of rotation axis of wind wheel $Z_{\mathrm{hub}}=15 \mathrm{~m}$.

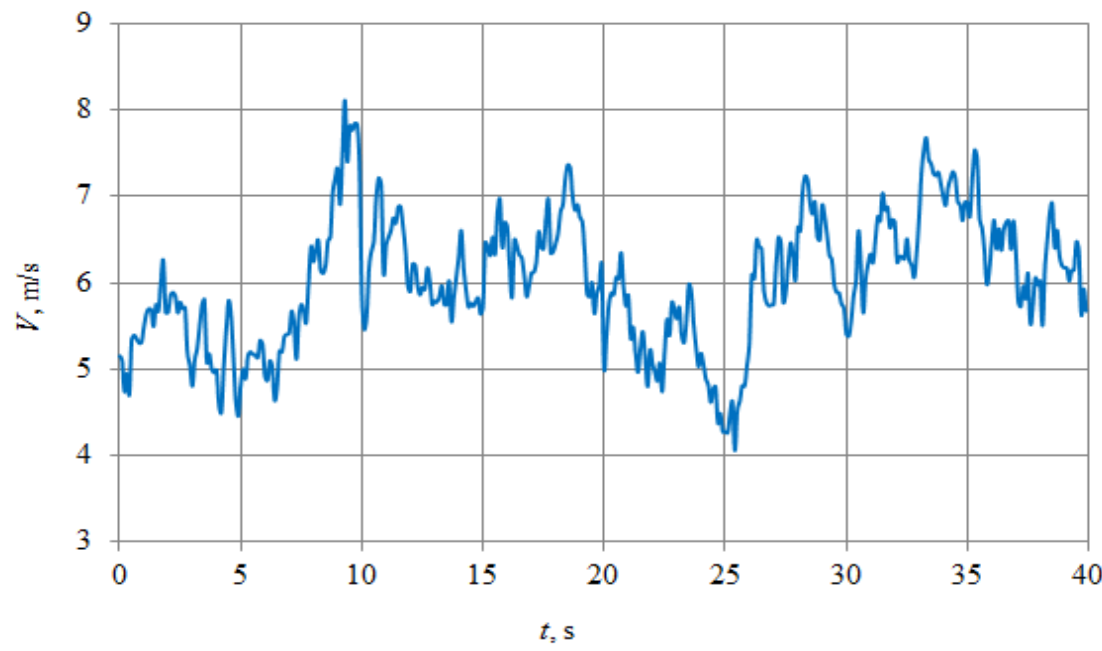

Fig.2. Results of simulation of the longitudinal component of wind speed

The simulation results are well consistent with the results of studies by other authors involved in the development of mathematical wind models for wind power [17-19].

A disadvantage of the above simulation method is that the spectral density $S(f)$ of the turbulent velocity component $v(\mathrm{t})$ is approximated by the sum of fractional-rational functions:

$$
S(f)=\sum_{k=1}^{N} S_{k}(f)
$$

that results in the need to create and store large number arrays in the RAM of the computer. 
A forming filter method can be used As an alternative method of simulation of random variable. According to this method, the value of the random variable $v(\mathrm{t})$ having the spectral density $S(f)$ will be equal to the output signal of the transfer function link $W(s)$ when exposed to the white noise $h(t)$ with a single spectral density when the condition is:

$$
S(f)=|W(s)|^{2}
$$

In practice, this method is implemented by selecting the family of frequency characteristics $W_{k}(s)$ at $k=1 \ldots N 2$, at which the approximate equality is achieved:

$$
S(f)=\sum_{k=1}^{N 2}\left|W_{k}(s)\right|^{2}
$$

It should be noted that the $N 2$ value is generally much smaller than $N$ in equation (6), which provides significant savings in computing resources.

\section{Implementation of dynamic wind model in MatLab/Simulink}

Computer implementation of wind model is carried out on the basis of $N 2$ statistically independent sources of white noise acting on $N 2$ of forming filters, output signals of which are summed. The authors of the [18-20] have obtained a satisfactory approximation to the initial spectral density for the Kaimal distribution at the number of generating filters $N 2=5$ in the frequency range up to $1 \mathrm{~Hz}$, which is quite acceptable for wind power calculations. Transfer functions for the Kaimal distribution are as follows [15]:

$$
\begin{aligned}
& W_{1}(s)=\frac{\sqrt{270 \cdot D \cdot L \cdot V} \cdot s}{\frac{3}{2 \pi} \cdot \frac{L}{V^{2}} \cdot s+0.6 L}, W_{2}(s)=\frac{\sqrt{2898 \cdot D \cdot \frac{L}{V}} \cdot s}{\frac{3}{2 \pi} \cdot \frac{L^{2}}{V^{2}} \cdot s^{2}+3.15 \cdot \frac{L}{V} \cdot s+1.2 \pi}, \\
& W_{3}(s)=\frac{\sqrt{6300 \cdot D \cdot \frac{L}{V}} \cdot s}{\frac{3}{2 \pi} \cdot \frac{L^{2}}{V^{2}} \cdot s^{2}+11.4 \cdot \frac{L}{V} \cdot s+21.7 \pi}, W_{4}(s)=\frac{\sqrt{9000 \cdot D \cdot \frac{L}{V}} \cdot s}{\frac{3}{2 \pi} \cdot \frac{L^{2}}{V^{2}} \cdot s^{2}+43.5 \cdot \frac{L}{V} \cdot s+315.4 \pi}, \\
& W_{5}(s)=\frac{\sqrt{13200 \cdot D \cdot \frac{L}{V}} \cdot s}{\frac{3}{2 \pi} \cdot \frac{L^{2}}{V^{2}} \cdot s^{2}+186 \cdot \frac{L}{V} \cdot s+5766 \pi} .
\end{aligned}
$$

In expression (15), the following variable designations are used: $D$ is the dispersion of the longitudinal component of wind speed, $\mathrm{m}^{2} / \mathrm{s}^{2}$; Lis the scale of the longitudinal component of atmospheric turbulence; $V$ is the average wind speed at the level of the wind wheel hub, $\mathrm{m} / \mathrm{s}$. The wind model implemented as a subsystem in MatLab/Simulink is shown in Figure 3.

The primary initial wind flow simulation data are:

$Z_{0}$ - roughness of the Earth's surface, m; $Z_{\text {hub }}$ - tower height of WT at the level of the hub, m; $V$ - the average wind speed accepted for simulation, $\mathrm{m} / \mathrm{s}$.

The calculation sequence for preparing input data to the model dialog form is as follows:

1. The value of the $Z_{0}$ is determined from Table 2 and the specified location of the WT.

2. Taking into account the recommendations [21] in Table 1, class of WT and intensity of turbulence $I_{\text {ref. }}$ are determined.

3. Equation (9) determines the standard deviation of the longitudinal component of wind speed $\sigma$ and its dispersion $D=\sigma^{2}$.

4. Equations (10), (11) determine the scale parameter of the longitudinal component of the speed vector $L$. 
Figure 4 shows an example of simulating of a longitudinal component of wind speed over a time interval of 40 seconds with initial simulation data corresponding to Figure 2.
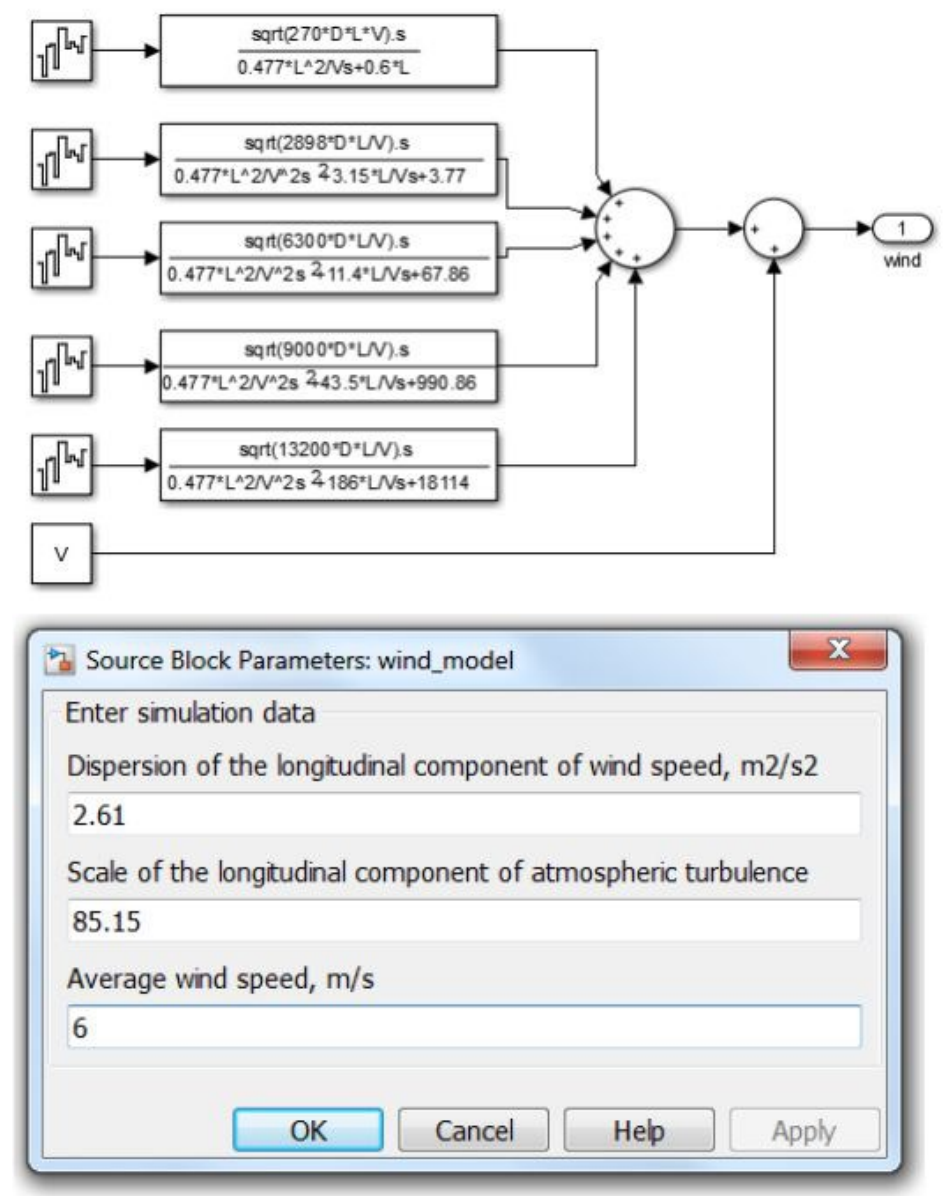

Fig. 3. Wind flow model with input parameters window

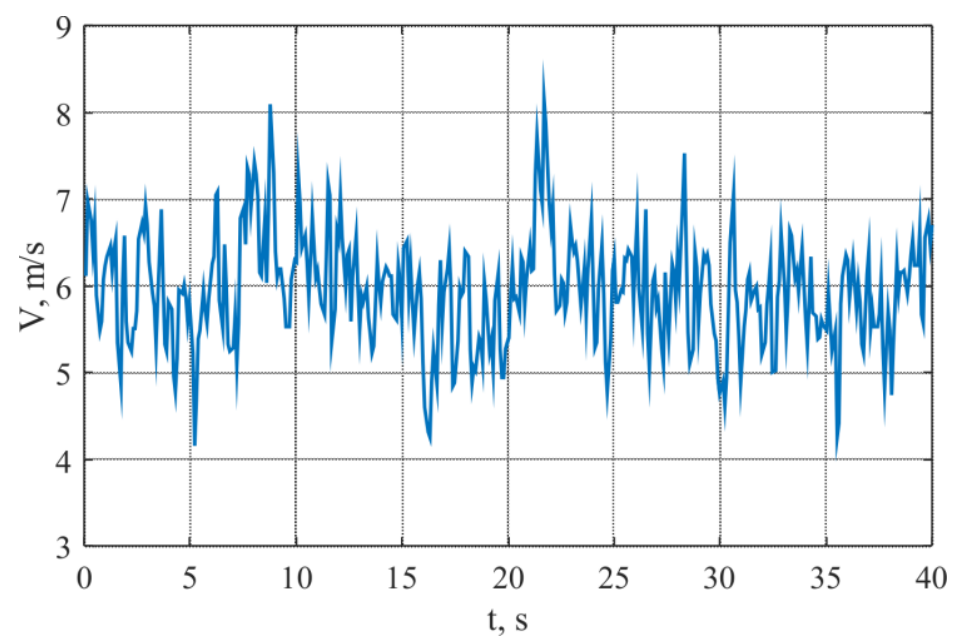

Fig. 4. Results of simulation of the longitudinal component of wind speed

The analysis of the obtained results shows that the wind flow model implemented on the basis of the method of forming filters provides adequate simulation of the longitudinal component of wind speed and can be used to solve the problems of wind power engineering. 


\section{Conclusion}

As a result of the research a dynamic wind model has been developed and implemented in the MatLab/Simulink software complex, which can be used to solve a wide range of wind power problems: research of wind turbine performance during turbulence and stochastic changes in wind speed, development of effective algorithms for control of the operating mode of the WT, etc. The model is implemented in the form of a separate functional unit, which provides simplicity and convenience of its practical application.

\section{Acknowledgements}

The study was supported by the Ministry of Education and Science of the Russian Federation. The unique identifier is RFMEFI57617X0098 (agreement No. 14.576.21.0098 dated 26 September 2017).

\section{REFERENCES}

1 Renewables 2018 Global Status Rep ort. Renewable Energy Policy Network for the 21 st Century. 2018. - 325 p. Available at: http://www.ren21.net/ (accessed 20 October 2019).

2 Cherubini A., Papini A., Vertechy R., Fontana M. Airborne Wind Energy Systems: A review of the technologies. Renewable and Sustainable Energy Reviews, 2015, Vol. 51, pp. 1461-1476.

3 Goudarzi N., Zhu W.D. A review on the development of wind turbine generators across the world. International Journal of Dynamics and Control, 2013, Vol. 1, No. 2, pp.192-202.

4 Abdullah M.A., Yatim A.H., Tan C.W., et al. A review of maximum power point tracking algorithms for wind energy systems. Renewable and Sustainable Energy Reviews, 2012, Vol. 16, pp. 3220-3227.

5 Guo R, DuJ., J. Wu, Y. Liu, The Pitch Control Algorithm of Wind Turbine Based on Fuzzy Control and PID Control. Energy and Power Engineering, 2013, Vol. 5, pp. 6-10.

6 Surzhikova O. Power supply of remote and almost inaccessible settlements. IOP Conference Series: Materials Science and Engineering IOP. 2015, Vol. 81, No 1 doi10.1088/1757-899X/81/1/012098

7 Bemš, J., Starý, O., Macaš, M., Žegklitz, J., Pošík, P. Innovative default prediction approach. Expert Systems with Applications, 2015, Vol. 42, No 17-18, pp. 6277-6285. doi: 10.1016/j.eswa.2015.04.053

8 Kerrouche K., Mezouar E., Boumediene A., et al. Modeling and Lyapunov-Designed based on Adaptive Gain Sliding Mode Control for Wind Turbines. Journal of Power Technologies, 2016, Vol. 96, No.2, pp.124-136.

9 Barbosa de Alencar D., De Mattos Affonso C., Limão de Oliveira R.C., et al. Different Models for Forecasting Wind Power Generation: Case Study. Energies, 2017, Vol. 10, No. 1976, pp. $1-27$.

10 Sarsikeev Y, Lukutin B.V, Lyapunov D.Y., Surkov M.A., Obuhov S.G. Dynamic model of wind speed longitudinal component. Advanced Materials Research, 2014, Vol. 953-954, pp. 529-532.

11 Abo-Khalil A.G., Alyami S., Sayed K., Alhejji A. Dynamic Modeling of Wind Turbines Based on Estimated Wind Speed under Turbulent Conditions. Energies, 2019, Vol. 12, No. 1907, pp. 1-25.

12 Yunus K., Thiringer T., Chen P. ARIMA-Based Frequency-Decomposed Modeling of Wind Speed Time Series. IEEE Transactions on Power System, 2016, Vol. 31, pp. 2546-2556.

13 Smilden E., Sørensen A., Eliassen L. Wind Model for Simulation of Thrust Variations on a Wind Turbine. Energy Procedia, 2016, Vol. 94, pp. 306 - 318.

14 IEC 61400-1: 2005 Wind Turbines - Part 1: Design requirements. Available at: https://webstore.ansi.org/Standards/IEC/IEC61400AmdEd2010?msclkid

15 Kirpichnikova I.M., Matveyenko O.V. Computer simulation of wind pulsation depending on time. International Scientific Journal for Alternative Energy and Ecology, 2010, Vol. 4, No. 81, pp. 54-59.

16 Neammanee B., Sirisumrannukul S., Chatratana S. Development of a Wind Turbine Simulator for Wind Generator Testing. International Energy Journal, 2007, Vol. 8, pp. 21 - 28.

17 Gavrilin A., Moyzes B., Kuvshinov K., Vedyashkin M., Surzhikova O. Determination of optimal milling modes by means of shock-vibration load reduction on tool and peak-factor equipment. Materials Science Forum, 2019, Vol. 942, pp. 87 - 96. doi:10.4028/www.scientific.net/MSF.942.87

18 Hao H., Gu B., Yan R., Hui H. Simulation and Analysis of Direct-driven Wind Turbine. International Journal of Online and Biomedical Engineering, 2015, Vol. 11, No. 5, pp. 17 - 23.

19 Sakipova S.E., Tanasheva N.K. Modeling aerodynamics of the wind turbine with rotating cylinders. Eurasian Physical Technical Journal. 2019, Vol.16, No. 1(31), pp. 88 - 93. DOI: 10.31489/2019No1/88-93

20 Králík T., Bemš, J., Starý, O. Electricity markets integrations - What is the current status and future outlook of bidding zones reconfiguration? Proc. of the $9^{\text {th }}$ Intern. Scient.Symposium on Electrical Power Engineering, ELEKTROENERGETIKA 2017, 237 - 240.

21 Erich Hau Wind Turbines: Fundamentals, Technologies, Application, Economics. 2-nd edition. Springer - Verlag Berlin Heidelberg. 2006, 783 p. 